Ворсанова С.Г..$^{1,2}$, Юров Ю.Б. ${ }^{1,2}$, Демидова И.А..,2, Колотий А.Д.,2, Куринная O.C. ${ }^{1,2}$, Кравец В.С., ${ }^{1,2}$, Юров И.Ю., $1,2,3$

БИОМАРКЕРЫ НЕОНКОЛОГИЧЕСКИХ БОЛЕЗНЕЙ МОЗГА, ОБУСЛОВЛЕННЫХ ХРОМОСОМНОЙ НЕСТАБИЛЬНОСТЬЮ, У ДЕТЕЙ

${ }^{1}$ Обособленное структурное подразделение «Научно-исследовательский клинический институт педиатрии имени академика Ю.Е. Вельтищева» Федерального государственного бюджетного образовательного учреждения высшего образования «Российский национальный исследовательский медицинский университет имени Н.И. Пирогова»,

117997, Россия, г. Москва, ул. Островитянова, 1

${ }^{2}$ Федеральное государственное бюджетное научное учреждение «научный центр психического здоровья»,

115522, Россия, г. Москва, Каширское ш., 34

${ }^{3}$ Федеральное государственное бюджетное образовательное учреждениедополнительного профессионального образования «Российская медицинская академия непрерывного профессионального образования»,

125993, Россия, г. Москва, ул. Барикадная, 2/1, стр.1

E-mail: ivan.iourov@gmail.com

Аннотация. Актуальность. За последнее десятилетие было предоставлено исчерпывающее количество данных о том, что хромосомная нестабильность является не только молекулярным и клеточным механизмом онкологических заболеваний, но также может быть причиной болезней мозга у детей. Цель исследования. В связи с вышесказанным, целью настоящей работы явилась критическая оценка распространенности хромосомной нестабильности у детей с нервнопсихическими заболеваниями для понимания механизмов ее возникновения, разработки тактики диагностики, как данной формы хромосомной патологии, так и геномных вариаций ее вызывающих. Maтериалы и методы. На основе цитогенетических и молекулярно-цитогенетических исследований 1000 детей с умственной отсталостью, аутизмом, эпилепсией и/или врожденными пороками развития был оценен потенциал анализа хромосомной нестабильности и биоинформатической оценки данных молекулярного кариотипирования для определения молекулярных (клеточных) механизмов заболевания. Результаты. Было обнаружено, что у $10.8 \%$ детей с вышеуказанными формами нервно-психических нарушений выявляется хромосомная нестабильность. Биоинформатический анализ позволил сделать вывод о том, что данная форма хромосомной патологии является результатом нарушения «молекулярный путей» (pathways) сохранности стабильности генома, репарации/репликации ДНК, а также регуляции клеточного цикла и запрограммированной гибели клеток за счет регулярных геномных вариаций. Заключение. Полученные данные свидетельствуют о том, что хромосомная нестабильность может быть биомаркером для неонкологических болезней мозга у детей. Более того, в настоящей работе предложен алгоритм идентификации молекулярных и клеточных механизмов заболеваний мозга у детей, обусловленных хромосомной нестабильностью. 
Ключевые слова: биомаркеры; болезни мозга; хромосомная нестабильность; хромосомные аномалии; молекулярное кариотипирование; биоинформатика; геномные вариации.

Информация для цитирования: Биомаркеры неонкологических болезней мозга, обусловленных хромосомной нестабильностью, у детей / С.Г. Ворсанова [и др.] // Научный результат. Медицина и фармация. 2018. T. 4, N 2. C. 8-17. DOI: 10.18413/2313-8955-2018-4-2-0-2

S.G. Vorsanova ${ }^{1,2}$, Yu.B. Yurov ${ }^{1,2}$, I.A. Demidova ${ }^{1,2}$, A.D. Kolotiy ${ }^{1,2}$, O.A. Kurinnaya ${ }^{1,2}$, V.S. Kravets ${ }^{1,2}$, I.Yu. Iourov ${ }^{1,2,3}$

\title{
BIOMARKERS FOR CHILDHOOD NONMALIGNANT BRAIN DISEASES ASSOCAITED WITH CHROMOSOME INSTABILITY
}

${ }^{1}$ Academician Y.E. Veltishchev Research Clinical Institute of Pediatrics, Pirogov Russian National Research Medical University,

1 Ostrovityanova St., Moscow, Russia, 117997

${ }^{2}$ Mental Health Research Center,

34 Kashirskoe Shosse, Moscow, Russia, 115522

${ }^{3}$ Russian Medical Academy of Continuous Postgraduate Education, build. 1, 2/1 Barikadnaya St., Moscow, Russia, 125993

E-mail: ivan.iourov@gmail.com

\begin{abstract}
Background. Over the past ten years, there has been provided a sufficient amount of data demonstrating that chromosome instability is not limited to being a molecular and cellular mechanism for oncologic diseases but may be also a cause of childhood brain diseases. The aim of the study. Thus, the study is aimed at the evaluation of chromosome instability occurrence in children with neuropsychiatric diseases for understanding the mechanism and for developing diagnostic tactics to uncover genomic variations causative for chromosome instability. Materials and methods. The article presents the cytogenetic, molecular cytogenetic and bioinformatics studies of 1000 children with intellectual disability, autism, epilepsy, and/or congenital malformations for uncovering molecular (cellular) mechanisms of the disease. Results. Chromosome instability is found to occur in $10.8 \%$ of children with the aforementioned diseases. Bioinformatics analysis has highlighted genomic alterations to genome stability maintenance, DNA reparation/replication, cell cycle, and programmed cell death pathways as a cause of this type of chromosomal pathology. Conclusion. Our data suggest that chromosome instability is a biomarker for nonmalignant brain diseases in children. Moreover, we propose an algorithm for identification of molecular and cellular mechanisms of childhood brain diseases resulted from chromosome instability.
\end{abstract}

Keywords: biomarkers; brain diseases; chromosome instability; chromosomal abnormalities; molecular karyotyping; bioinformatics; genomic variations.

Information for citation: Vorsanova SG, Yurov YuB, Demidova IA, et al. Biomarkery neonkologicheskikh bolezney mozga, obuslovlennykh khromosomnoy nestabil'nost'yu, u detey [Biomarkers for childhood nonmalignant brain diseases asso- 
caited with chromosome instability]. Research Result. Medicine and Pharmacy. 2018;4(2):8-17. DOI: 10.18413/2313-8955-2018-4-2-0-2

Введение. Современные достижения в области медицинской генетики позволили обнаружить широкий спектр геномной патологии, ассоциированной с умственной отсталостью, аутизмом, эпилепсией и/или врожденными пороками развития [2, 4, 25]. Особое место среди различных форм генетических нарушений, ассоциированных с патологией центральной нервной системы, занимает хромосомная нестабильность. В настоящее время известно, что эта форма соматической вариабельности генома на хромосомном уровне является одним из распространенных механизмов болезней, вызванных нарушением развития центральной нервной системы, а также нейродегенеративных заболеваний $[1,4,9,15]$. Более того, ранее было высказано предположение о том, что некоторые формы хромосомной нестабильности (например, спорадическая анеуплоидия и неспецифические структурные хромосомные аномалии) могут быть использованы в качестве маркеров для идентификации аномальных процессов (каскадов процессов), приводящих к патологии центральной нервной системы $[15,24]$.

В современной литературе геномная (хромосомная) нестабильность преимущественно рассматривается в онкологическом контексте, являясь как одним из основных механизмов широко распространенных жизнеугрожающих раковых заболеваний, так и надежным биомаркером малигнизации клеточных популяций $[4,11,17,19,21]$. Тем не менее, известно, что хромосомная нестабильность может быть одним из ключевых элементов патогенетического каскада молекулярных и клеточных процессов, связанных с нарушениями репродукции [19], почечной патологией [16], нервными и психическими болезнями $[15,22]$, а также причиной различных изменений психики, вызванных отрицательным воздействием окружающей среды на организм человека [18]. Помимо этого, предполагается, что подобные изменения соматического генома могут быть одной из причин старения организма на тканевом и клеточным уровнях [23]. Однако, анализируя работы, посвященные хромосомной нестабильности, можно сделать обоснованный вывод о том, что она практически некогда не рассматривалась в контексте биомаркеров болезней мозга, несмотря на значимую роль в патогенезе нарушений центральной нервной системы [1, 4, 15, 22, 24]. Биомаркеры представляют собой изменения в тканях, клетках или биологических жидкостях организма, характерные для определенного патологического состояния, которые могут быть объективно и воспроизводимо измерены с помощью методов клеточной или молекулярной биологии $[8,20]$. Поскольку хромосомная нестабильность полностью соответствует данному определению, ее оценка в качестве биомаркера аномальных процессов, приводящих к нарушению функционирования центральной нервной системы у детей, является обоснованной.

Цель исследования. Целью настоящего исследования явилась оценка потенциала использования наличия различных форм хромосомной нестабильности в качестве биомаркеров неонкологических болезней мозга у детей при молекулярной и клеточной диагностике геномной патологии с целью идентификации механизмов патогенеза заболевания.

Материалы и методы. Тысяча детей с умственной отсталостью, аутизмом, эпилепсией и/или врожденными пороками развития были исследованы с помощью цитогенетических методов G- и С-окрашивания [3]. При цитогенетическом анализе хромосомной нестабильности исследовалось не менее 30 метафазных пластинок (клеток) для каждого пациента. Молекулярноцитогенетическое исследование проводилось методом флюоресцентной гибридизации in situ (FISH) с использованием ориги- 
нальных хромосомоспецифичных ДНК проб $[1,3,4]$ и включало в себя анализ 100 метафазных пластинок и 1000 интерфазных ядер для каждого исследованного пациента. Данные результатов молекулярного кариотипирования (разрешение сканирования генома не менее 1 тыс. пн) были обработаны с использованием оригинального биоинформатического метода оценки функциональных последствий геномных вариаций в виде изменения числа копий последовательностей ДНК и геномной нестабильности $[4,15]$, основанный на исследовании межтканевой вариабельности экспрессии генов, вовлеченных в перестройку in silico, интерактомном анализе белков, кодируемых этими генами, и идентификации возможных изменений «молекулярных путей» (pathways), вызванных регулярными (наследуемыми или de novo) геномными вариациями.

Результаты и обсуждение. У 108 детей с умственной отсталостью, аутизмом, эпилепсией и/или врожденными пороками развития (10.8\%) была обнаружена хромосомная нестабильность, которая проявлялась в виде анеуплоидии, крупных структурных перестроек (транслокации, делеции, дупликации, инверсии), ломкости хромосом и хромосомных разрывов. В случаях проявления хромосомной нестабильности в виде анеуплоидии была использована FISH с оригинальными хромосомоспецифичными ДНК пробами. Хромосомная нестабильность в виде мносомии и трисомии была подтверждена. В 31-ом случае было использовано молекулярное кариотипирования в сочетании с оригинальным биоинформатическим методом поиска геномных вариаций, которые могли бы быть причиной хромосомной нестабильности. Во всех исследованных случаях были выявлены вариации числа копий последовательностей ДНК в количестве от 4 до 9 на каждый геном, которые имеют функциональные последствия для таких «молекулярных путей» (pathways), как сохранность стабильности генома, репарация/репликация ДНК, регуляция клеточного цикла и запрограммированной гибели клеток. Согласно литературным дан- ным [4, 5, 12, 15, 17], подобные нарушения вышеуказанных «кластеров» молекулярных и клеточных процессов приводят к хромосомной нестабильности. Таким образом, учитывая полученные данные и предыдущие исследования $[1,4,6,12-15,26]$, можно сделать вывод о том, что в описываемых случаях умственной отсталости, аутизма, эпилепсии и врожденных пороков развития хромосомная нестабильность является одним из ключевых элементов патогенетического каскада заболевания и, как следствие, может быть признана механизмом возникновения фенотипических проявлений. Примечательно, что изменения «молекулярных путей» регуляции клеточного цикла и запрограммированной гибели клеток, в основном, ассоциировались с такими формами хромосомной нестабильности, как анеуплоидия. Геномные вариации, связанные с модифицирующим эффектом «молекулярных путей» сохранности стабильности генома и репликации/репарации ДНК были преимущественно обнаружены при нестабильности, проявляющейся в виде крупных структурных перестроек, ломкости хромосом и хромосомных разрывов. Следовательно, обоснован вывод о том, что различные типы хромосомной нестабильности могут служить биомаркерами аномальных процессов, приводящих к нарушению функционирования центральной нервной системы у детей.

В настоящее время известно большее число геномных вариаций, приводящих к хромосомной нестабильности [4, 5, 17]. В частности, нарушения определенных молекулярных и клеточных процессов, ассоциированных с регуляцией сохранности стабильности генома и репликации/репарации ДНК, могут быть причиной ломкости хромосом [7, 10]. Примечательно, что в настоящей работе были выявлены вариации числа копий последовательностей ДНК, затрагивающие гены, которые вовлечены в соответствующие «молекулярные пути», у детей с ломкостью хромосом. Наименее изученными формами хромосомной нестабильности с точки зрения причин их возникновения являются тканеспецифические измене- 
ния числа, нарушения морфологии и разрывы хромосом. Тем не менее, в литературе имеются данные, позволяющие выделить ряд процессов-кандидатов возникновения этих форм хромосомной нестабильности, которые входят в «молекулярные пути» сохранности стабильности генома, репарацию/репликацию ДНК, регуляцию клеточного цикла и запрограммированной гибели клеток $[1,14,15,26]$. В связи с вышесказанным, видится необходимым учитывать регулярные геномные вариации, которые ассоциированы с хромосомной нестабильностью, при молекулярной диагностике с целью определения механизма заболевания.

Вариабельность соматического генома является одним из основных механизмов генетической вариабельности на клеточном, тканевом и межиндивидуальном уровнях [6]. Примечательно, что изменения структуры и поведения генома в соматических клетках являются также элементом патогенетического каскада процессов при психических и нейродегенеративных заболеваниях [1-4, 22]. Более того, изменения структуры и морфологии хромосом могут быть результатом отрицательного воздействия окружающей среды, приводя к функциональным нарушениям различных систем организма человека, включая центральную нервную систему $[12,13,18]$. Следовательно, помимо определения причинно-следственной связи между фактом наличия хромосомной нестабильности и вариабельностью генома, имеет значение также мониторинг и более детальный цитогенетический (молекулярно-цитогенетический) анализ типов изменения структуры, числа и морфологии хромосом. К сожалению, на сегодняшний день подобный подход к диагностическим исследованиям хромосомной нестабильности крайне редок.

Учитывая потенциальную диагностическую ценность анализа особенностей и причин возникновения хромосомной нестабильности, являющейся биомаркером патологических процессов при нервнопсихических болезнях у детей, было признано целесообразным пересмотреть общепри- нятую последовательность действий при идентификации хромосомных аномалий. Цитогенетический анализ и молекулярное кариотипирование обычно рассматриваются, как два последовательных этапа диагностики хромосомных аномалий, несмотря на то, что массивы данных, полученных с помощью каждого из этих методов, являются взаимодополняющими, а не иерархическими (например, нередко ошибочно утверждается, что данные цитогенетического анализа вторичны по отношению к результатам молекулярного кариотипирования, или же диагностика хромосомной патологии сводится к проведению исключительно молекулярного кариотипирования) [4, 24]. Проведенное исследование указывает на то, что для увеличения эффективности диагностики хромосомных аномалий за счет идентификации механизма заболевания требуется ряд дополнений. В частности, цитогенетический анализ целесообразно дополнить цитогенетическими и молекулярно-цитогенетическими исследованиями генома в большем числе клеток, регистрируя все типы изменения структуры, числа и морфологии хромосом, а молекулярное кариотипирование, в свою очередь, дополнить биоинформатической оценкой последствий регулярных (структурных) геномных вариаций в контексте их возможного эффекта на «молекулярные пути» сохранности стабильности генома, репарации/репликации ДНК регуляции клеточного цикла и запрограммированной гибели клеток. В результате внедрения в протоколы диагностики хромосомной (геномной) патологии у детей предлагаемых дополнений алгоритм идентификации молекулярных и клеточных механизмов заболевания, обусловленного хромосомной нестабильностью, будет выглядеть следующим образом: параллельно получается два массива данных: (1) цитогенетические и молекулярноцитогенетические данные о характере хромосомной нестабильности; (2) данные молекулярного кариотипирования; далее, с помощью биоинформатического анализа второго массива и корреляции его с первым получается возможность определения причины 


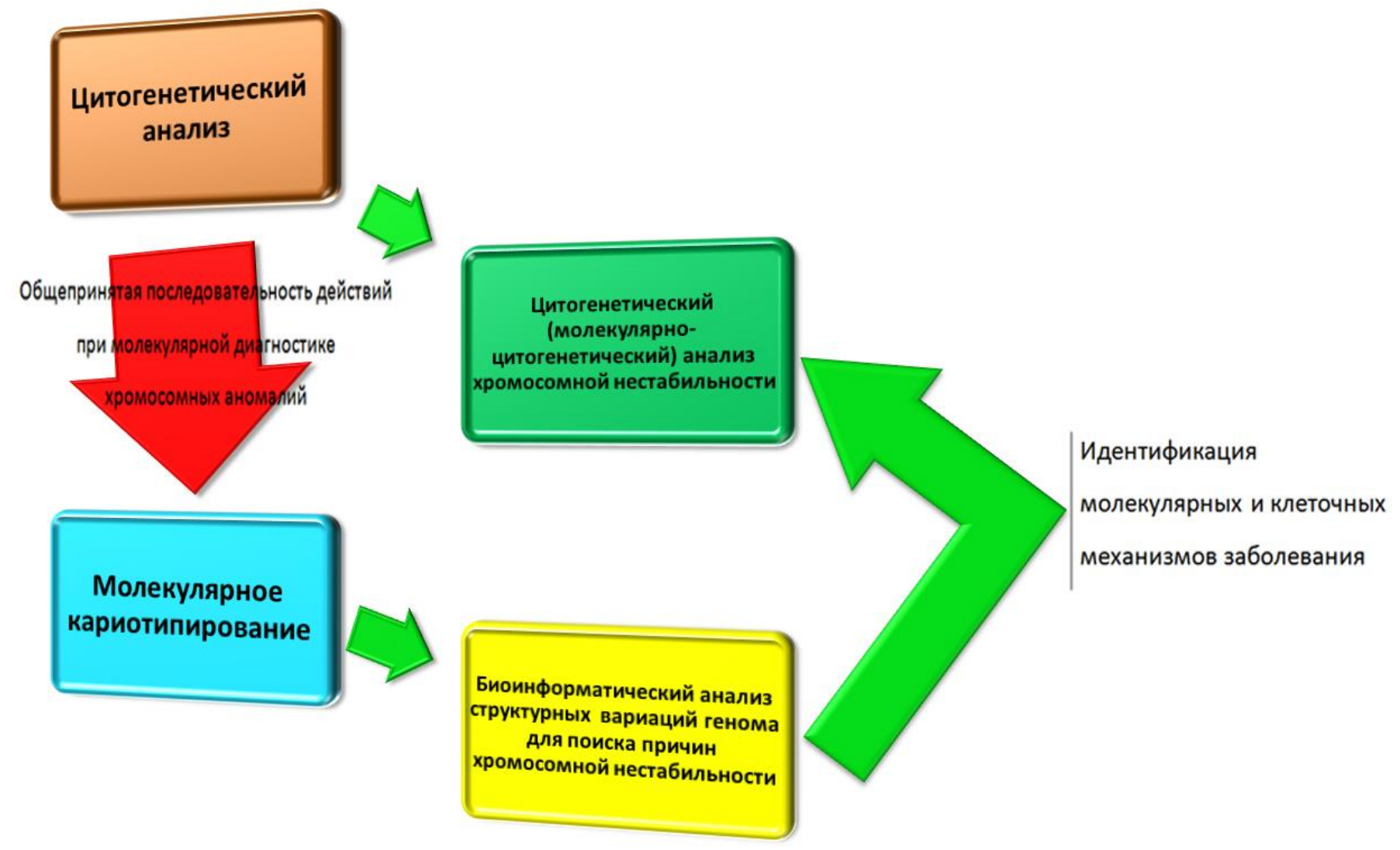

Рис. Схематическое изображение алгоритма идентификации молекулярных и клеточных механизмов заболевания,

обусловленного хромосомной нестабильностью. В отличие от общепринятой последовательности действий при молекулярной диагностике хромосомной патологии рекомендуется проводить более детальный анализ хромосомной нестабильности и определение ее возможных причин по данным молекулярного кариотипирования

Fig. Schematic representation of the algorithm for identification of molecular and cellular mechanisms of the disease caused by chromosomal instability. In contrast to the generally accepted sequence of actions for molecular diagnostics of chromosomal pathology, it is recommended to conduct a more detailed analysis of chromosomal instability and to determine its possible causes according to data of molecular karyotyping 
хромосомной нестабильности, в частности, и механизма заболевания, в целом. На рисунке схематически изображен предлагаемый алгоритм идентификации молекулярных и клеточных механизмов заболевания, обусловленного хромосомной нестабильностью.

Заключение. У детей с умственной отсталостью, аутизмом, эпилепсией и/или врожденными пороками развития хромосомная нестабильность, выявляемая с помощью цитогенетического анализа, наблюдается в не менее чем $10 \%$ случаев. По данным биоинформатического анализа результатов молекулярного кариотипирования хромосомная нестабильность является результатом нарушения «молекулярный путей» (pathways) сохранности стабильности генома, репарации/репликации ДНК, регуляции клеточного цикла и запрограммированной гибели клеток. Суммируя результаты цитогенетического, молекулярноцитогенетического и биоинформатического анализа, показано, что различные формы хромосомной нестабильности могут рассматриваться в качестве биомаркеров аномальных процессов, приводящих к нарушению функционирования центральной нервной системы у детей.

Изменения процессов сохранности стабильности генома, репарации/репликации ДНК, клеточного цикла и запрограммированной гибели клеток на молекулярном и клеточном уровнях вызваны, в свою очередь, регулярными геномными вариациями, модифицирующими соответствующие «молекулярные пути». Таким образом, обоснован вывод о том, что общепринятая последовательность действий при диагностике хромосомной патологии, которая ограничивается проведением цитогенетического анализа и последующего молекулярного кариотипирования, требует существенной доработки.

В настоящей работе предложено рассматривать цитогенетические анализ и молекулярное кариотипирование не как два последовательных диагностических исследования, а как две взаимодополняющие процедуры, необходимые для идентификации не только хромосомной патологии, но и механизма заболевания. Для реализации этой идеи предлагается цитогенетический анализ дополнить цитогенетическими/молекулярно-цитогенетическими исследованиями хромосомной нестабильности, а молекулярное кариотипирование - биоинформатической оценкой последствий геномных вариаций в контексте их возможного эффекта на «молекулярные пути» сохранности стабильности генома, репарации/репликации ДНК, регуляции клеточного цикла и запрограммированной гибели клеток. Сочетание цитогенетических, геномных и постгеномных/интепретационных технологий обладает большим потенциалом для молекулярной и клеточной диагностики с последующей разработкой тактики лечения и прогнозирования течения заболеваний у детей, обусловленных хромосомной нестабильностью.

В отношении данной статьи не было зарегистрировано конфликта интересов.

\section{Список литературы}

1. Нестабильность хромосом в нервных клетках человека в норме и при нервнопсихических заболеваниях / Ю.Б. Юров [и др.] // Генетика. 2010. Т. 46, N 10. С. 1352-1355.

2. Современные представления о молекулярной генетике и геномике аутизма / С.Г. Ворсанова [и др.] // Фундаментальные Исследования. 2013. N 4. Часть 2. С. 356-367.

3. Цитогенетические, молекулярноцитогенетические и клинико-генеалогические исследования матерей детей с аутизмом: поиск семейных генетических маркеров аутистических расстройств / С.Г. Ворсанова [и др.] // Журнал Неврологии и Психиатрии им. С.С. Корсакова. 2009. T. 109, N 6. C. 54-64.

4. Юров И.Ю., Ворсанова С.Г., Юров Ю.Б. Геномные и хромосомные болезни центральной нервной системы: молекулярные и цитогенетические аспекты. М.: Медпрактика-М, 2014. $384 \mathrm{c}$.

5. Aguilera A., García-Muse T. Causes of genome instability // Annual Reviewers of Genetics. 2013. Vol. 47. P. 1-32.

6. Astolfi P.A., Salamini F., Sgaramella V. Are we Genomic Mosaics? Variations of the Genome of Somatic Cells can Contribute to Diversify our Phenotypes // Current Genomics. 2010. Vol. 11(6). P. 379-386.

7. Bjerregaard V.A., Özer Ö., Hickson I.D., Liu Y. The Detection and Analysis of Chromosome 
Fragile Sites // Methods in Molecular Biology. 2018. Vol. 1672. P. 471-482.

8. Bonassi S., Au W.W. Biomarkers in molecular epidemiology studies for health risk prediction // Mutation Research. 2002. Vol. 511(1). P. 73-86.

9. Coppedè F., Migliore L. DNA damage in neurodegenerative diseases // Mutation Research. 2015. Vol. 776. P. 84-97.

10.Debatisse M., Le Tallec B., Letessier A., Dutrillaux B., Brison O. Common fragile sites: mechanisms of instability revisited // Trends in Genetics. 2012. Vol. 28(1). P. 22-32.

11.Fenech M. Chromosomal biomarkers of genomic instability relevant to cancer // Drug Discovery Today. 2002. Vol. 7(22). P. 1128-1137.

12.Heng H.H., Liu G., Stevens J.B., Abdallah B.Y., Horne S.D., Ye K.J., Bremer S.W., Chowdhury S.K., Ye C.J. Karyotype heterogeneity and unclassified chromosomal abnormalities // Cytogenetic and Genome Research. 2013. Vol. 139(3). P. 144-157.

13.Heng H.H., Regan S.M., Liu G., Ye C.J. Why it is crucial to analyze non clonal chromosome aberrations or NCCAs? // Molecular Cytogenetics. 2016. Vol. 9. Article. 15. 12 p.

14.Horne S.D., Chowdhury S.K., Heng H.H. Stress, genomic adaptation, and the evolutionary trade-off // Frontiers in Genetics. 2014. Vol. 5. Article. 92. $6 \mathrm{p}$.

15.Iourov I.Y., Vorsanova S.G., Liehr T., Kolotii A.D., Yurov Y.B. Increased chromosome instability dramatically disrupts neural genome integrity and mediates cerebellar degeneration in the ataxia-telangiectasia brain // Human Molecular Genetics. 2009. Vol. 18(14). P. 2656-2669.

16.Khan Z., Pandey M., Samartha R.M. Role of cytogenetic biomarkers in management of chronic kidney disease patients: A review // International Journal of Health Sciences (Qassim). 2016. Vol. 10(4). P. 576-589.

17.Liu G., Stevens J.B, Horne S.D., Abdallah B.Y., Ye K.J., Bremer S.W., Ye C.J., Chen D.J., Heng H.H. Genome chaos: survival strategy during crisis // Cell Cycle. 2014. Vol. 13(4). P. 528-537.

18.Liu G., Ye C.J., Chowdhury S.K., Abdallah B.Y., Horne S.D., Nichols D., Heng H.H. Detecting chromosome condensation defects in gulf war illness patients // Current Genomics. 2018. Vol. 19(3). P. 200-206.

19.Mandrioli D., Belpoggi F., Silbergeld E.K., Perry M.J. Aneuploidy: a common and early evidence-based biomarker for carcinogens and reproductive toxicants // Environmental Health. 2016. Vol. 15(1). Article. 97. 10 p.
20.Mayeux R. Biomarkers: potential uses and limitations // NeuroRx. 2004. Vol. 1(2). P. 182-188.

21.Peterson S.E., Loring J.F. Genomic instability in pluripotent stem cells: implications for clinical applications // The Journal of Biological Chemistry. 2014. Vol. 289(8). P. 4578-4584.

22.Smith C.L., Bolton A., Nguyen G. Genomic and epigenomic instability, fragile sites, schizophrenia and autism // Current Genomics. 2010. Vol. 11(6). P. 447-469.

23.Vijg J. Somatic mutations, genome mosaicism, cancer and aging // Current Opinion in Genetics \& Development. 2014. Vol. 26. P. 141-149.

24.Vorsanova S.G., Yurov Y.B., Soloviev I.V., Iourov I.Y. Molecular cytogenetic diagnosis and somatic genome variations // Current Genomics. 2010. Vol. 11(6). P. 440-446.

25.Waye M.M.Y., Cheng H.Y. Genetics and epigenetics of autism: A Review // Psychiatry and Clinical Neurosciences. 2018. Vol. 72(4). P. 228-244.

26.Ye C.J., Regan S., Liu G., Alemara S., Heng H.H. Understanding aneuploidy in cancer through the lens of system inheritance, fuzzy inheritance and emergence of new genome systems // Molecular Cytogenetics. 2018. Vol. 11. Article. 31. $13 \mathrm{p}$.

\section{References}

1. Yurov YB, Vorsanova SG, Solov'ev IV, Iourov IYu. Instability of chromosomes in human nerve cells (normal and with neuromental diseases). Russian Journal of Genetics. 2010;46(10):11941196.

2. Vorsanova SG, Yurov YB, Silvanovich AP, Demidova IA, Iourov IYu. Sovremennyye predstavleniya o molekulyarnoy genetike i genomike autizma [Modern ideas about molecular genetics and genomics of autism]. Fundamental Research. 2013;4-2:356-367. Russian.

3. Vorsanova SG, Voinova VYu, Iourov IYu, Kurinnaya OS, Demidova IA, Yurov YB. Tsitogeneticheskiye, molekulyarno-tsitogeneticheskiye i kliniko-genealogicheskiye issledovaniya materey detey s autizmom: poisk semeynykh geneticheskikh markerov autisticheskikh rasstroystv [Cytogenetic, molecular cytogenetic and clinical genealogy studies of mothers of children with autism: the search for family genetic markers of autistic disorders]. Zhurnal nevrologii i psikhiatrii imeni S.S. Korsakova. 2009;109(6):54-64. Russian.

4. Iourov IYu, Vorsanova SG, Yurov YB. Genomnyye i khromosomnyye bolezni tsentral'noy nervnoy sistemy: molekulyarnyye i tsitogeneticheskiye aspekty [Genomic and chromosomal diseas- 
es of the central nervous system: molecular and cytogenetic aspects]. Moscow: Medpraktika-M; 2014. 384 p. Russian.

5. Aguilera A, García-Muse T. Causes of genome instability. Annual Reviewers of Genetics. 2013;47:1-32.

6. Astolfi PA, Salamini F, Sgaramella V. Are we Genomic Mosaics? Variations of the Genome of Somatic Cells can Contribute to Diversify our Phenotypes. Current Genomics. 2010;11(6):379-386.

7. Bjerregaard VA, Özer Ö, Hickson ID, Liu Y. The Detection and Analysis of Chromosome Fragile Sites. Methods in Molecular Biology. 2018; 1672:471-482.

8. Bonassi $\mathrm{S}, \mathrm{Au} \mathrm{WW}$. Biomarkers in molecular epidemiology studies for health risk prediction. Mutation Research. 2002;511(1):73-86.

9. Coppedè F, Migliore L. DNA damage in neurodegenerative diseases. Mutation Research. 2015;776:84-97.

10.Debatisse M, Le Tallec B, Letessier A, Dutrillaux B, Brison O. Common fragile sites: mechanisms of instability revisited. Trends in Genetics. 2012;28(1):22-32.

11.Fenech M. Chromosomal biomarkers of genomic instability relevant to cancer. Drug Discovery Today. 2002;7(22):1128-1137.

12.Heng HH, Liu G, Stevens JB, Abdallah BY, Horne SD, Ye KJ, Bremer SW, Chowdhury SK, Ye CJ. Karyotype heterogeneity and unclassified chromosomal abnormalities. Cytogenetic and Genome Research. 2013;139(3):144-157.

13.Heng HH, Regan SM, Liu G, Ye CJ. Why it is crucial to analyze non clonal chromosome aberrations or NCCAs? Molecular Cytogenetics. 2016;9. Article 15. 12 p.

14.Horne SD, Chowdhury SK, Heng HH. Stress, genomic adaptation, and the evolutionary trade-off. Frontiers in Genetics. 2014; 5. Article 92. $6 \mathrm{p}$.

15.Iourov IY, Vorsanova SG, Liehr T, Kolotii AD, Yurov YB. Increased chromosome instability dramatically disrupts neural genome integrity and mediates cerebellar degeneration in the ataxiatelangiectasia brain. Human Molecular Genetics. 2009;18(14):2656-2669.

16.Khan Z, Pandey M, Samartha RM. Role of cytogenetic biomarkers in management of chronic kidney disease patients: A review. International Journal of Health Sciences (Qassim). 2016;10(4):576-589.

17.Liu G, Stevens JB, Horne SD, Abdallah BY, Ye KJ, Bremer SW, Ye CJ, Chen DJ, Heng
HH. Genome chaos: survival strategy during crisis. Cell Cycle. 2014;13(4):528-537.

18.Liu G, Ye CJ, Chowdhury SK, Abdallah BY, Horne SD, Nichols D, Heng HH. Detecting chromosome condensation defects in gulf war illness patients. Current Genomics. 2018;19(3): 200-206.

19.Mandrioli D, Belpoggi F, Silbergeld EK, Perry MJ. Aneuploidy: a common and early evidence-based biomarker for carcinogens and reproductive toxicants. Environmental Health. 2016;15(1). Article 97. 10 p.

20.Mayeux R. Biomarkers: potential uses and limitations. NeuroRx. 2004;1(2):182-188.

21.Peterson SE, Loring JF. Genomic instability in pluripotent stem cells: implications for clinical applications. The Journal of Biological Chemistry. 2014;289(8):4578-4584.

22.Smith CL, Bolton A, Nguyen G. Genomic and epigenomic instability, fragile sites, schizophrenia and autism. Current Genomics. 2010;11(6):447-469.

23.Vijg J. Somatic mutations, genome mosaicism, cancer and aging. Current Opinion in Genetics \& Development. 2014;26:141-149.

24.Vorsanova SG, Yurov YB, Soloviev IV, Iourov IY. Molecular cytogenetic diagnosis and somatic genome variations. Current Genomics. 2010;11(6):440-446.

25.Waye MMY, Cheng HY. Genetics and epigenetics of autism: A Review. Psychiatry and Clinical Neurosciences. 2018;72(4):228-244.

26. Ye CJ, Regan S, Liu G, Alemara S, Heng $\mathrm{HH}$. Understanding aneuploidy in cancer through the lens of system inheritance, fuzzy inheritance and emergence of new genome systems. Molecular Cytogenetics. 2018;11. Article 31. 13 p.

Ворсанова Светлана Григорьевна, доктор биологических наук, профессор, заслуженный деятель науки РФ, академик РАЕ, заведующая лабораторией.

Юров Юрий Борисович, доктор биологических наук, профессор, заслуженный деятель науки РФ, академик РАЕ, заведующий лабораторией.

Демидова Ирина Александровна, кандидат биологических наук, ведущий научный сотрудник.

Колотий Алексей Дмитриевич, кандидат биологических наук, старший научный сотрудник.

Куринная Оксана Сергеевна, кандидат биологических наук, научный сотрудник.

Кравец Виктор Сергеевич, кандидат биологических наук, научный сотрудник. 
Юров Иван Юрьевич, доктор биологических наук, профессор РАН, заведующий лабораторией.

Vorsanova Svetlana Grigorievna, Doctor of Biological Sciences, Professor, Honored Scientist of Russia, Academician of the Russian Academy of Natural Sciences, Head of the Laboratory.

Yurov Yuri Borisovich, Doctor of Biological Sciences, Professor, Honored Scientist of Russia, Academician of the Russian Academy of Natural Sciences, Head of the Laboratory.

Demidova Irina Aleksandrovna, Candidate of Biological Sciences, Leading Researcher.
Kolotiy Alexey Dmitrievich, Candidate of Biological Sciences, Senior Researcher.

Kurinnaya Oksana Sergeevna, Candidate of Biological Sciences, Researcher.

Kravets Victor Sergeevich, Candidate of Biological Sciences, Researcher.

Iourov Ivan Yurievich, Doctor of

Biological Sciences, Professor of the Russian

Academy of Sci-ences, Head of Laboratory.

Статья поступила в редакцию 12 марта 2018 г. 
DOI: 10.18413/2313-8955-2018-4-2-0-3

Данилко К.В. ${ }^{1}$, Назарова Л.Ш., Хабибуллина P.P.1, Хайруллина P.P.', Слесаренко Я.С. ${ }^{1}$, Шамиева М.В. ${ }^{2}$, Муталова Э.Г.', Викторова Т.В. ${ }^{1,3}$
ПОЛИМОРФИЗМ ГЕНОВ IL10, CTLA4 И ПРЕДРАСПОЛОЖЕННОСТЬ К РЕВМАТОИДНОМУ АРТРИТУ

${ }^{1}$ Федеральное государственное бюджетное образовательное учреждение высшего образования «Башкирский государственный медицинский университет», 450008, Россия, г. Уфа, ул. Ленина, 3

${ }^{2}$ Федеральное государственное бюджетное образовательное учреждение высшего образования «Башкирский государственный педагогический университет им. М. Акмуллы», 450008, Россия, г. Уфа, ул. Октябрьской революции, 3А

${ }^{3}$ Федеральное государственное бюджетное учреждение науки Институт биохимии и генетики Уфимского научного центра Российской академии наук 450054, Россия,

г. Уфа, просп. Октября, 71

E-mail: kse-danilko@yandex.ru

Аннотация. Актуальность. Ревматоидный артрит (РА) - это системное аутоиммунное заболевание, сопровождающееся хроническим воспалением синовиальной оболочки суставов и широким спектром внесуставных проявлений. Проблема ранней диагностики и выявления новых маркеров РА остается на сегодняшний день весьма актуальной. Цель исследования. Целью данной работы был поиск ассоциаций вариантов полиморфных локусов $-592 \mathrm{C}>\mathrm{A}$ гена ILIO и +6230G>A гена CTLA4 с развитием PA у жителей Республики Башкортостан. Материалы и методы. В исследование было включено 204 пациента с РА и 324 индивида контрольной группы из Республики Башкортостан. Изучение полиморфных локусов генов ILIO и CTLA4 проводилось методом ПЦР в реальном времени, а статистическая обработка результатов - с использованием критерия $\chi^{2}$ и оценкой отношения шансов (OR) с 95\% доверительным интервалом (CI). Результаты. При исследовании полиморфного локуса -592C>A гена ILIO было установлено, что генотип СС служит маркером повышенного риска развития РА $(\mathrm{p}=0.042, \mathrm{OR}=1.467,95 \% \mathrm{CI} 1.015-2.122)$, в то время как генотип СА ассоциирован со снижением риска заболевания ( $\mathrm{p}=0.017$, OR=0.634, 95\% CI 0.436-0.922). После стратификации согласно этнической принадлежности обе ассоциации оказались характерны для представителей башкирского этноса (CC: $\mathrm{p}=0.036$, $\mathrm{OR}=3.221,95 \%$ CI 1.070-9.879 и СA: $\mathrm{p}=0.008$, OR=0.224, 95\%CI 0.071-0.692). Различия между пациентами с РА и индивидами контрольной группы русской этнической принадлежности достигли уровня статистической значимости только по генотипу CA ( $\mathrm{p}=0.027$, OR=0.470, 95\%CI 0.239-0.923). У татар распределение частот генотипов и аллелей полиморфного локуса $-592 \mathrm{C}>\mathrm{A}$ гена ILIO в группе пациентов с РА и в контрольной группе оказалось статистически неотличимым $(\mathrm{p}>0.8)$. Для вариантов полиморфного локуса $+6230 \mathrm{G}>\mathrm{A}$ гена CTLA4 ассоциаций с развитием РА не наблюдалось. Заключение. Таким образом, в данной работе выявлена взаимосвязь вариантов полиморфного локуса -592C >A гена ILIO с развитием РА у жителей Республики Башкортостан (с учетом этнических особенностей). Доказательств наличия существенного вклада полиморфизма $+6230 \mathrm{G}>\mathrm{A}$ гена CTLA4 в формирование предрасположенности к РА не установлено. 